CARPATHIAN J. MATH.

Volume 38 (2022), No. 2,

Pages 503 - 516

\title{
Extragradient method with a new adaptive step size for solving non-Lipschitzian pseudo-monotone variational inequalities
}

\author{
DUONG VIET THONG
}

\begin{abstract}
The purpose of this work is to develop a new version of the extragradient method for solving non-Lipschitzian and pseudo-monotone variational inequalities in real Hilbert spaces. First, we prove a sufficient condition for weak convergence of a proposed algorithm under relaxed assumptions. Next, under strong pseudomonotonicity and Lipschitz continuity assumptions, we obtain also a Q-linear convergence rate of this algorithm. Our results improve some recent contributions in the literature on the extragradient method.
\end{abstract}

\section{INTRODUCTION}

We are focus on the following classical variational inequality (VI) $([10,11])$ which consists in finding a point $x^{*} \in C$ such that

$$
\left\langle F x^{*}, x-x^{*}\right\rangle \geq 0 \forall x \in C,
$$

where $C$ is a nonempty closed convex subset in a real Hilbert space $H$, and $F: C \rightarrow H$ is a single-valued mapping. As commonly done, we denote by $\operatorname{Sol}(C, F)$ the solution set of VI (1.1). Variational inequalities are fundamental in a broad range of mathematical and applied sciences; the theoretical and algorithmic foundations as well as the applications of variational inequalities have been extensively studied in the literature and continue to attract intensive research. For the current state of the art in finite dimensional setting, see for instance [9] and the extensive list of references therein.

Many authors have proposed and analyzed several iterative methods for solving the variational inequality (1.1). The simplest one is the following projection method, which can be seen an extension of the projected gradient method introduced for solving optimization problems.

$$
x_{n+1}=P_{C}\left(x_{n}-\tau F x_{n}\right),
$$

for each $n \geq 1$, where $P_{C}$ denotes by the metric projection from $H$ onto $C$. It is shown that the convergence of this method is quite restrictive and assume that $F$ is $L$-Lipschitz continuous and $\alpha$-strongly monotone (will be explained in the sequel), and $\tau \in\left(0, \frac{2 \alpha}{L^{2}}\right)$.

To avoid the hypothesis of the strong monotonicity, Korpelevich [20] (also independently by [1]) proposed a double projection method known as the extragradient method in Euclidean space when $F$ is monotone and $L$-Lipschitz continuous. The iterative step of the method is a $\mathrm{s}$ follows.

$$
x_{0} \in C, y_{n}=P_{C}\left(x_{n}-\tau_{n} F x_{n}\right), x_{n+1}=P_{C}\left(x_{n}-\tau_{n} F y_{n}\right),
$$

where $\tau_{n} \in\left(0, \frac{1}{L}\right)$.

Received: 06.02.2021. In revised form: 05.01.2022. Accepted: 12.01.2022

2010 Mathematics Subject Classification. 47H09, 47J20, 47J05, 47J25.

Key words and phrases. Extragradient method, variational inequality, pseudomonotone operator, convergence rate. 
In recent years, the extragradient method was further extended to infinite-dimensional spaces in various ways, see, e.g. $[6,3,4,5,12,15,19,21,22,25,24,23,28,29]$ and the references therein.

However, when the inequality variational mapping is not Lipschitz continuous or its Lipschitz constant $L$ is difficult to compute/approximate, Korpelevich's method fails since the step-size $\tau_{n}$ depends on this. Indeed, we give an academic example of a variational inequality problem in the infinite dimensional spaces which shows that the mapping is pseudo-monotone and uniformly continuous on $C$ but it fails to be Lipschitz continuous on $C$ with the solution set of the variational inequality mapping is nonempty. Hence, the method (1.2) cannot be applied.

Example 1.1. Consider the Hilbert space

$$
H=l_{2}:=\left\{u=\left.\left(u_{1}, u_{2}, \ldots, u_{n}, \ldots\right)\left|\sum_{n=1}^{\infty}\right| u_{n}\right|^{2}<+\infty\right\}
$$

equipped with the inner product and induced norm on $H$ :

$$
\langle u, v\rangle=\sum_{n=1}^{\infty} u_{n} v_{n} \text { and }\|u\|=\sqrt{\langle u, u\rangle}
$$

for any $u=\left(u_{1}, u_{2}, \ldots, u_{n}, \ldots\right), v=\left(v_{1}, v_{2}, \ldots, v_{n}, \ldots\right) \in H$.

Consider the set and the mapping:

$$
C=\{u \in H \mid\|u\| \leq \alpha\}, \quad F u=\left((\|u\|+\alpha)-\frac{1}{\|u\|+\alpha}\right) u
$$

where $\alpha>1$ is a positive real number.

With this $C$ and $F$, it is easy to see that $\operatorname{Sol}(C, F)=\{0\}$ and moreover, $F$ is pseudomonotone and uniformly continuous on $C$ but $F$ fails to be Lipschitz continuous on $C$.

First observe that since $\alpha>1$, we get that

$$
\left((\|u\|+\alpha)-\frac{1}{\|u\|+\alpha}\right)>0 \forall u \in C .
$$

Now let $u, v \in C$ be such that $\langle F u, v-u\rangle \geq 0$. This implies that $\langle u, v-u\rangle \geq 0$.

Consequently,

$$
\begin{aligned}
\langle F v, v-u\rangle & =\left((\|u\|+\alpha)-\frac{1}{\|u\|+\alpha}\right)\langle v, v-u\rangle \\
& \geq\left((\|u\|+\alpha)-\frac{1}{\|u\|+\alpha}\right)(\langle v, v-u\rangle-\langle u, v-u\rangle) \\
& =\left((\|u\|+\alpha)-\frac{1}{\|u\|+\alpha}\right)\|v-u\|^{2} \geq 0
\end{aligned}
$$

meaning that $F$ is pseudo-monotone.

Now, since $C$ is compact, the mapping $F$ is uniformly continuous on $C$.

Finally we show that $F$ is not Lipschitz continuous on $C$. Assume to the contrary that $F$ is Lipschitz continuous on $C$, i.e., there exists $L>0$ such that

$$
\|F u-F v\| \leq L\|u-v\| \forall u, v \in C .
$$

Let $u=(L, 0, \ldots, 0, \ldots)$ and $v=(0,0, \ldots, 0, \ldots)$ then

$$
\|F u-F v\|=\|F u\|=\left((\|u\|+\alpha)-\frac{1}{\|u\|+\alpha}\right)\|u\|=\left((L+\alpha)-\frac{1}{L+\alpha}\right) L .
$$


Extragradient method with a new adaptive step size for solving non-Lipschitzian pseudo-monotone variational inequalitie85

Thus, $\|F u-F v\| \leq L\|u-v\|$ is equivalent to

$$
\left((L+\alpha)-\frac{1}{L+\alpha}\right) L \leq L^{2}
$$

equivalently

$$
L+\alpha \leq L+\frac{1}{L+\alpha}<L+1
$$

which implies that $\alpha<1$, and this leads to a contraction and thus $F$ is not Lipschitz continuous on $C$.

Motivated by Example 1.1, it would be of interest to propose an iterative method for solving $\operatorname{Sol}(C, F)$ for which the underline cost function $F$ is uniformly continuous on bounded subsets of $C$ but not Lipschitz continuous on $C$. Iusem [16] proposed the following algorithm so that it can solve this problem.

\section{Algorithm 1.}

Initialization: Given $\gamma>0, l \in(0,1), \mu \in(0,1)$. Let $x_{0} \in C$ be arbitrary

Iterative Steps: Given the current iterate $x_{n}$, calculate $x_{n+1}$ as follows:

Step 1. Compute

$$
y_{n}=P_{C}\left(x_{n}-\tau_{n} F x_{n}\right)
$$

where $\tau_{n}:=\gamma l^{m_{n}}$ and $m_{n}$ is the smallest non-negative integer $m$ satisfying

$$
\gamma l^{m}\left\|F x_{n}-P_{C}\left(x_{n}-\gamma l^{m} F x_{n}\right)\right\| \leq \mu\left\|x_{n}-P_{C}\left(x_{n}-\gamma l^{m} F x_{n}\right)\right\| .
$$

If $x_{n}=y_{n}$ then stop and $x_{n}$ is a solution of $\operatorname{Sol}(C, F)$. Otherwise

Step 2. Compute

$$
x_{n+1}=P_{C}\left(x_{n}-\beta_{n} F y_{n}\right),
$$

where

$$
\beta_{n}:=\frac{\left\langle F y_{n}, x_{n}-y_{n}\right\rangle}{\left\|F y_{n}\right\|^{2}}
$$

Set $n:=n+1$ and go to Step 1 .

Recently, Cai et al. [2] constructed a modification of the subgradient extragradient method [3] which converges under a weaker condition in Hilbert spaces. To be more specific, they assumed that $F$ is uniformly continuous pseudo-monotone operator and proved a strong convergence theorem under some appropriate conditions imposed on the parameters. In particular, they introduced the algorithm which uses a different Armijotype line-search and then $F$ is only assumed to be pseudo-monotone on $C$ in the sense of Karamardian [18]. In this paper, motivated by the works of Cai et al., we propose a new extragradient method for solving variational inequality problems of pseudo-monotone and non-Lipschitz continuous operator in real Hilbert spaces. We present weak convergence and convergence rate of the proposed algorithm in real Hilbert spaces under suitable conditions. Our results improve some recent contributions in the literature.

The paper is organized as follows. We first recall some basic definitions and results in Section 2. Our algorithms are presented and analysed in Section 3. 


\section{PRELIMINARIES}

Let $H$ be a real Hilbert space and $C$ be a nonempty, closed and convex subset of $H$. The weak convergence of $\left\{x_{n}\right\}_{n=1}^{\infty}$ to $x$ is denoted by $x_{n} \rightarrow x$ as $n \rightarrow \infty$, while the strong convergence of $\left\{x_{n}\right\}_{n=1}^{\infty}$ to $x$ is written as $x_{n} \rightarrow x$ as $n \rightarrow \infty$. For each $x, y \in H$ we have

$$
\|x+y\|^{2} \leq\|x\|^{2}+2\langle y, x+y\rangle \text {. }
$$

Definition 2.1. Let $T: H \rightarrow H$ be an operator.

(1) The operator $T$ is called $L$-Lipschitz continuous with $L>0$ if

$$
\|T x-T y\| \leq L\|x-y\| \quad \forall x, y \in H .
$$

(2) The operator $T$ is called monotone if

$$
\langle T x-T y, x-y\rangle \geq 0 \quad \forall x, y \in H .
$$

(3) The operator $T$ is called pseudo-monotone if

$$
\langle T x, y-x\rangle \geq 0 \Longrightarrow\langle T y, y-x\rangle \geq 0 \quad \forall x, y \in H .
$$

(4) The operator $T$ is called $\alpha$-strongly monotone if there exists a constant $\alpha>0$ such that

$$
\langle T x-T y, x-y\rangle \geq \alpha\|x-y\|^{2} \forall x, y \in H .
$$

(5) The operator $T$ is called sequentially weakly continuous if for each sequence $\left\{x_{n}\right\}$ we have: $\left\{x_{n}\right\}$ converges weakly to $x$ implies $T x_{n}$ converges weakly to $T x$.

It is easy to see that general monotone operator is pseudo-monotone but the converse is not true.

For every point $x \in H$, there exists a unique nearest point in $C$, denoted by $P_{C} x$ such that $\left\|x-P_{C} x\right\| \leq\|x-y\| \forall y \in C$. $P_{C}$ is called the metric projection of $H$ onto $C$. It is known that $P_{C}$ is nonexpansive.

Lemma 2.1. ([14]) Let $C$ be a nonempty closed convex subset of a real Hilbert space $H$. Given $x \in H$ and $z \in C$. Then $z=P_{C} x \Longleftrightarrow\langle x-z, z-y\rangle \geq 0 \forall y \in C$.

Lemma 2.2. ([14]) Let $C$ be a closed and convex subset in a real Hilbert space $H, x \in H$. Then

i) $\left\|P_{C} x-P_{C} y\right\|^{2} \leq\left\langle P_{C} x-P_{C} y, x-y\right\rangle \forall y \in C$;

ii) $\left\|P_{C} x-y\right\|^{2} \leq\|x-y\|^{2}-\left\|x-P_{C} x\right\|^{2} \forall y \in C$;

iii) $\left\langle\left(I-P_{C}\right) x-\left(I-P_{C}\right) y, x-y\right\rangle \geq\left\|\left(I-P_{C}\right) x-\left(I-P_{C}\right) y\right\|^{2} \forall y \in C$.

For properties of the metric projection, the interested reader could be referred to [14, Section 3].

The following Lemmas are useful for the convergence of our proposed methods.

Lemma 2.3. ([8]) For $x \in H$ and $\alpha \geq \beta>0$ the following inequalities hold.

$$
\begin{gathered}
\frac{\left\|x-P_{C}(x-\alpha A x)\right\|}{\alpha} \leq \frac{\left\|x-P_{C}(x-\beta A x)\right\|}{\beta}, \\
\left\|x-P_{C}(x-\beta A x)\right\| \leq\left\|x-P_{C}(x-\alpha A x)\right\| .
\end{gathered}
$$

Lemma 2.4. ([17]) Let $H_{1}$ and $H_{2}$ be two real Hilbert spaces. Suppose $A: H_{1} \rightarrow H_{2}$ is uniformly continuous on bounded subsets of $H_{1}$ and $M$ is a bounded subset of $H_{1}$. Then $A(M)$ (the image of $M$ under $A$ ) is bounded. 
Extragradient method with a new adaptive step size for solving non-Lipschitzian pseudo-monotone variational inequalitie8

Lemma 2.5. [7, Lemma 2.1] Consider the problem $V I(C, A)$ with $C$ being a nonempty, closed, convex subset of a real Hilbert space $H$ and $A: C \rightarrow H$ being pseudo-monotone and continuous. Then, $x^{*}$ is a solution of $\operatorname{VI}(C, A)$ if and only if

$$
\left\langle A x, x-x^{*}\right\rangle \geq 0 \forall x \in C .
$$

Lemma 2.6. ([26]) Let $C$ be a nonempty set of $H$ and $\left\{x_{n}\right\}$ be a squence in $H$ such that the following two conditions hold:

i) for every $x \in C, \lim _{n \rightarrow \infty}\left\|x_{n}-x\right\|$ exists;

ii) every sequential weak cluster point of $\left\{x_{n}\right\}$ is in $C$.

Then $\left\{x_{n}\right\}$ converges weakly to a point in $C$.

Definition 2.2. [27] Let $\left\{x_{n}\right\}$ be a sequence in $H$.

i) $\left\{x_{n}\right\}$ is said to converge R-linearly to $x^{*}$ with rate $\rho \in[0,1)$ if there is a constant $c>0$ such that

$$
\left\|x_{n}-x^{*}\right\| \leq c \rho^{n} \forall n \in \mathbb{N} .
$$

ii) $\left\{x_{n}\right\}$ is said to converge $Q$-linearly to $x^{*}$ with rate $\rho \in[0,1)$ if

$$
\left\|x_{n+1}-x^{*}\right\| \leq \rho\left\|x_{n}-x^{*}\right\| \forall n \in \mathbb{N} .
$$

\section{MAIN RESULTS}

In this section, we introduce the new modification of extragradient method solving the VI (1.1). We present the weak convergence and convergence rate of the sequence generated by the proposed method under the suitable assumptions. The proposed algorithm is of the form:

Algorithm 2. Given $\gamma>0, l, \mu \in(0,1)$. Let $v_{0} \in C$ be arbitrary. Compute

$$
\begin{aligned}
& y_{n}=P_{C}\left(v_{n}-\tau_{n} F v_{n}\right), \\
& v_{n+1}=P_{C}\left(v_{n}-\tau_{n} F y_{n}\right),
\end{aligned}
$$

with $\tau_{n}:=\gamma l^{m_{n}}$ where $m_{n}$ is the smallest nonnegative integer $m$ satisfying

$\gamma l^{m}\left\langle F P_{C}\left(v_{n}-\gamma l^{m} F v_{n}\right)-F v_{n}, P_{C}\left(v_{n}-\gamma l^{m} F v_{n}\right)-P_{C}\left(v_{n}-\gamma l^{m} F P_{C}\left(v_{n}-\gamma l^{m} F v_{n}\right)\right\rangle\right.$

$$
\leq \frac{\mu}{2}\left(\left\|v_{n}-P_{C}\left(v_{n}-\gamma l^{m} F v_{n}\right)\right\|^{2}+\left\|P_{C}\left(v_{n}-\gamma l^{m} F v_{n}\right)-P_{C}\left(v_{n}-\gamma l^{m} P_{C}\left(v_{n}-\gamma l^{m} F v_{n}\right)\right)\right\|^{2}\right) .
$$

Remark 3.1. (3.3) can rewritten as follows

$$
\gamma l^{m}\left\langle F y_{n}-F v_{n}, y_{n}-v_{n+1}\right\rangle \leq \frac{\mu}{2}\left(\left\|v_{n}-y_{n}\right\|^{2}+\left\|y_{n}-v_{n+1}\right\|^{2}\right) .
$$

3.1. Weak convergence. To prove the weak convergence we need the following conditions:

Condition 1. The feasible set $C$ of the VI (1.1) is a nonempty, closed, and convex subset of the real Hilbert space $H$.

Condition 2. The operator $F: C \rightarrow H$ is a pseudo-monotone, uniformly continuous on bounded subsets of $C$. Moreover, the mapping $F: C \rightarrow H$ satisfies the following condition

$$
\text { whenever }\left\{x_{n}\right\} \subset C, x_{n} \rightarrow z \text {, one has }\|F z\| \leq \liminf _{n \rightarrow \infty}\left\|F x_{n}\right\| \text {. }
$$


Condition 3. The solution set of the VI (1.1) is nonempty, that is $\operatorname{Sol}(C, F) \neq \emptyset$.

We start the algorithm's convergence analysis by proving that (3.3) terminates after finite steps.

Lemma 3.7. Assume that the mapping $F: C \rightarrow H$ is uniformly continuous on bounded subsets of $C$. The Armijo-line search rule (3.3) is well defined. In addition, we have $\tau_{n} \leq \gamma$.

Proof. If $v_{n} \in \operatorname{Sol}(C, F)$ then $v_{n}=P_{C}\left(v_{n}-\gamma F v_{n}\right)$ and $m_{n}=0$. We consider the situation $v_{n} \notin \operatorname{Sol}(C, F)$ and assume the contrary that for all $m$ we have

$\gamma l^{m}\left\langle F P_{C}\left(v_{n}-\gamma l^{m} F v_{n}\right)-F v_{n}, P_{C}\left(v_{n}-\gamma l^{m} F v_{n}\right)-P_{C}\left(v_{n}-\gamma l^{m} F P_{C}\left(v_{n}-\gamma l^{m} F v_{n}\right)\right\rangle\right.$

$>\frac{\mu}{2}\left(\left\|v_{n}-P_{C}\left(v_{n}-\gamma l^{m} F v_{n}\right)\right\|^{2}+\left\|P_{C}\left(v_{n}-\gamma l^{m} F v_{n}\right)-P_{C}\left(v_{n}-\gamma l^{m} P_{C}\left(v_{n}-\gamma l^{m} F v_{n}\right)\right)\right\|^{2}\right)$.

By Cauchy-Schwartz inequality, we have

$\gamma l^{m}\left\langle F P_{C}\left(v_{n}-\gamma l^{m} F v_{n}\right)-F v_{n}, P_{C}\left(v_{n}-\gamma l^{m} F v_{n}\right)-P_{C}\left(v_{n}-\gamma l^{m} F P_{C}\left(v_{n}-\gamma l^{m} F v_{n}\right)\right\rangle\right.$

$\leq \gamma l^{m}\left\|F P_{C}\left(v_{n}-\gamma l^{m} F v_{n}\right)-F v_{n}\right\| \| P_{C}\left(v_{n}-\gamma l^{m} F v_{n}\right)-P_{C}\left(v_{n}-\gamma l^{m} F P_{C}\left(v_{n}-\gamma l^{m} F v_{n}\right) \|\right.$,

and

$\left(\left\|v_{n}-P_{C}\left(v_{n}-\gamma l^{m} F v_{n}\right)\right\|^{2}+\left\|P_{C}\left(v_{n}-\gamma l^{m} F v_{n}\right)-P_{C}\left(v_{n}-\gamma l^{m} P_{C}\left(v_{n}-\gamma l^{m} F v_{n}\right)\right)\right\|^{2}\right)$

$\geq 2\left\|v_{n}-P_{C}\left(v_{n}-\gamma l^{m} F v_{n}\right)\right\|\left\|P_{C}\left(v_{n}-\gamma l^{m} F v_{n}\right)-P_{C}\left(v_{n}-\gamma l^{m} P_{C}\left(v_{n}-\gamma l^{m} F v_{n}\right)\right)\right\|$.

Combining (3.5) and (3.6) and (3.7) we find

$$
\gamma l^{m}\left\|F P_{C}\left(v_{n}-\gamma l^{m} F v_{n}\right)-F v_{n}\right\|>\mu\left\|P_{C}\left(v_{n}-\gamma l^{m} F v_{n}\right)-v_{n}\right\| .
$$

This implies that

$$
\left\|F v_{n}-F P_{C}\left(v_{n}-\gamma l^{m} F v_{n}\right)\right\|>\mu \frac{\left\|v_{n}-P_{C}\left(v_{n}-\gamma l^{m} F v_{n}\right)\right\|}{\gamma l^{m}} .
$$

Since $v_{n} \in C$ for all $n$ and $P_{C}$ is continuous, we have $\lim _{m \rightarrow \infty}\left\|v_{n}-P_{C}\left(v_{n}-\gamma l^{m} F v_{n}\right)\right\|=0$. From the uniform continuity of the operator $F$ on bounded subsets of $C$ it implies that

$$
\lim _{m \rightarrow \infty}\left\|F v_{n}-F P_{C}\left(v_{n}-\gamma l^{m} F v_{n}\right)\right\|=0 .
$$

Combining (3.9) and (3.10) we get

$$
\lim _{m \rightarrow \infty} \frac{\left\|v_{n}-P_{C}\left(v_{n}-\gamma l^{m} F v_{n}\right)\right\|}{\gamma l^{m}}=0 .
$$

Assume that $z_{m}=P_{C}\left(x_{n}-\gamma l^{m} F v_{n}\right)$ we have

$$
\left\langle z_{m}-v_{n}+\gamma l^{m} F v_{n}, x-z_{m}\right\rangle \geq 0 \forall x \in C .
$$

This implies that

$$
\left\langle\frac{z_{m}-v_{n}}{\gamma l^{m}}, x-z_{m}\right\rangle+\left\langle F v_{n}, x-z_{m}\right\rangle \geq 0 \forall x \in C .
$$

Taking the limit $m \rightarrow \infty$ in (3.12) and using (3.11) we obtain

$$
\left\langle F v_{n}, x-v_{n}\right\rangle \geq 0 \forall x \in C,
$$

which implies that $v_{n} \in \operatorname{Sol}(C, F)$ this is a contraction. 
Extragradient method with a new adaptive step size for solving non-Lipschitzian pseudo-monotone variational inequalitie8 9

Lemma 3.8. Assume that Conditions 1-3 hold and let $\left\{v_{n}\right\}$ be any sequence generated by Algorithm 2. If there exists a subsequence $\left\{v_{n_{k}}\right\}$ of $\left\{v_{n}\right\}$ such that $\left\{v_{n_{k}}\right\}$ converges weakly to $z \in C$ and $\lim _{k \rightarrow \infty}\left\|v_{n_{k}}-y_{n_{k}}\right\|=0$ then $z \in \operatorname{Sol}(C, F)$.

Proof. We have $y_{n_{k}}=P_{C}\left(v_{n_{k}}-\tau_{n_{k}} F v_{n_{k}}\right)$ thus,

$$
\left\langle v_{n_{k}}-\tau_{n_{k}} F v_{n_{k}}-y_{n_{k}}, x-y_{n_{k}}\right\rangle \leq 0 \forall x \in C \text {. }
$$

or equivalently

$$
\frac{1}{\tau_{n_{k}}}\left\langle v_{n_{k}}-y_{n_{k}}, x-y_{n_{k}}\right\rangle \leq\left\langle F v_{n_{k}}, x-y_{n_{k}}\right\rangle \forall x \in C .
$$

This implies that

$$
\frac{1}{\tau_{n_{k}}}\left\langle v_{n_{k}}-y_{n_{k}}, x-y_{n_{k}}\right\rangle+\left\langle F v_{n_{k}}, y_{n_{k}}-v_{n_{k}}\right\rangle \leq\left\langle F v_{n_{k}}, x-v_{n_{k}}\right\rangle \forall x \in C .
$$

Now, we show that

$$
\liminf _{k \rightarrow \infty}\left\langle F v_{n_{k}}, x-v_{n_{k}}\right\rangle \geq 0 \text {. }
$$

For showing this, we consider two possible cases. Suppose first that $\liminf _{k \rightarrow \infty} \tau_{n_{k}}>$ 0 . We have $\left\{v_{n_{k}}\right\}$ is a bounded sequence, $F$ is uniformly continuous on bounded subsets of $C$. By Lemma 2.5, we get that $\left\{F v_{n_{k}}\right\}$ is bounded. Taking $k \rightarrow \infty$ in (3.13) since $\left\|v_{n_{k}}-y_{n_{k}}\right\| \rightarrow 0$, we get

$$
\liminf _{k \rightarrow \infty}\left\langle F v_{n_{k}}, x-v_{n_{k}}\right\rangle \geq 0 .
$$

Now, we assume that $\lim \inf _{k \rightarrow \infty} \tau_{n_{k}}=0$. Assume $z_{n_{k}}=P_{C}\left(v_{n_{k}}-\tau_{n_{k}} \cdot l^{-1} F v_{n_{k}}\right)$, we have $\tau_{n_{k}} l^{-1}>\tau_{n_{k}}$. Applying Lemma 2.3, we obtain

$$
\left\|v_{n_{k}}-z_{n_{k}}\right\| \leq \frac{1}{l}\left\|v_{n_{k}}-y_{n_{k}}\right\| \rightarrow 0 \text { as } k \rightarrow \infty .
$$

Consequently, $z_{n_{k}} \rightarrow z \in C$, this implies that $\left\{z_{n_{k}}\right\}$ is bounded, which the uniformly continuity of the operator $A$ on bounded subsets of $C$ it follows that

$$
\left\|F v_{n_{k}}-F z_{n_{k}}\right\| \rightarrow 0 \text { as } k \rightarrow \infty .
$$

By the Armijo line-search rule (3.3) and the proof is similar to the inequality (3.8) we have

$$
\tau_{n_{k}} \cdot l^{-1}\left\|F v_{n_{k}}-F P_{C}\left(v_{n_{k}}-\tau_{n_{k}} l^{-1} F v_{n_{k}}\right)\right\|>\mu\left\|v_{n_{k}}-P_{C}\left(v_{n_{k}}-\tau_{n_{k}} l^{-1} F v_{n_{k}}\right)\right\| .
$$

That is,

$$
\frac{1}{\mu}\left\|F v_{n_{k}}-F z_{n_{k}}\right\|>\frac{v_{n_{k}}-z_{n_{k}} \|}{\tau_{n_{k}} l^{-1}} .
$$

Combining (3.15) and (3.16) we obtain

$$
\lim _{k \rightarrow \infty} \frac{\left\|v_{n_{k}}-z_{n_{k}}\right\|}{\tau_{n_{k}} l^{-1}}=0
$$

Furthermore, we have

$$
\left\langle v_{n_{k}}-\tau_{n_{k}} l^{-1} F v_{n_{k}}-z_{n_{k}}, x-z_{n_{k}}\right\rangle \leq 0 \forall x \in C .
$$

This implies that

$$
\frac{1}{\tau_{n_{k}} l^{-1}}\left\langle v_{n_{k}}-z_{n_{k}}, x-z_{n_{k}}\right\rangle+\left\langle F v_{n_{k}}, z_{n_{k}}-v_{n_{k}}\right\rangle \leq\left\langle F v_{n_{k}}, x-v_{n_{k}}\right\rangle \forall x \in C .
$$

Taking the limit $k \rightarrow \infty$ in (3.17) we get

$$
\liminf _{k \rightarrow \infty}\left\langle F v_{n_{k}}, x-v_{n_{k}}\right\rangle \geq 0 .
$$


Therefore, the inequality (3.14) is proved. Next, we show that $z \in \operatorname{Sol}(C, F)$.

Now we choose a sequence $\left\{\epsilon_{k}\right\}$ of positive numbers decreasing and tending to 0 . For each $k$, we denote by $N_{k}$ the smallest positive integer such that

$$
\left\langle F v_{n_{j}}, x-v_{n_{j}}\right\rangle+\epsilon_{k} \geq 0 \forall j \geq N_{k},
$$

where the existence of $N_{k}$ follows from (3.14). Since $\left\{\epsilon_{k}\right\}$ is decreasing, it is easy to see that the sequence $\left\{N_{k}\right\}$ is increasing. Furthermore, for each $k$, since $\left\{v_{N_{k}}\right\} \subset C$ we have $F v_{N_{k}} \neq 0$ and, setting

$$
u_{N_{k}}=\frac{F v_{N_{k}}}{\left\|F v_{N_{k}}\right\|^{2}},
$$

we have $\left\langle F v_{N_{k}}, v_{N_{k}}\right\rangle=1$ for each $k$. Now, we can deduce from (3.18) that for each $k$

$$
\left\langle F v_{N_{k}}, x+\epsilon_{k} u_{N_{k}}-v_{N_{k}}\right\rangle \geq 0 .
$$

Since the fact that $F$ is pseudo-monotone, we get

$$
\left\langle F\left(x+\epsilon_{k} u_{N_{k}}\right), x+\epsilon_{k} u_{N_{k}}-v_{N_{k}}\right\rangle \geq 0 .
$$

This implies that

$$
\left\langle F x, x-v_{N_{k}}\right\rangle \geq\left\langle F x-F\left(x+\epsilon_{k} u_{N_{k}}\right), x+\epsilon_{k} u_{N_{k}}-v_{N_{k}}\right\rangle-\epsilon_{k}\left\langle F x, u_{N_{k}}\right\rangle .
$$

Now, we show that $\lim _{k \rightarrow \infty} \epsilon_{k} u_{N_{k}}=0$. Indeed, we have $v_{n_{k}} \rightarrow z$ as $k \rightarrow \infty$. Since $F$ satisfies the condition (3.4). We have

$$
0<\|F z\| \leq \liminf _{k \rightarrow \infty}\left\|F_{n_{k}}\right\| \text { (note that } F z \neq 0 \text { otherwise, } z \text { is a solution). }
$$

Since $\left\{v_{N_{k}}\right\} \subset\left\{v_{n_{k}}\right\}$ and $\epsilon_{k} \rightarrow 0$ as $k \rightarrow \infty$, we obtain

$$
0 \leq \limsup _{k \rightarrow \infty}\left\|\epsilon_{k} u_{N_{k}}\right\|=\limsup _{k \rightarrow \infty}\left(\frac{\epsilon_{k}}{\left\|F v_{n_{k}}\right\|}\right) \leq \frac{\limsup _{k \rightarrow \infty} \epsilon_{k}}{\liminf _{k \rightarrow \infty}\left\|F v_{n_{k}}\right\|}=0,
$$

which implies that $\lim _{k \rightarrow \infty} \epsilon_{k} u_{N_{k}}=0$.

Now, letting $k \rightarrow \infty$, then the right hand side of (3.19) tends to zero by $F$ is uniformly continuous, $\left\{v_{N_{k}}\right\},\left\{u_{N_{k}}\right\}$ are bounded and $\lim _{k \rightarrow \infty} \epsilon_{k} u_{N_{k}}=0$. Thus, we get

$$
\liminf _{k \rightarrow \infty}\left\langle F x, x-v_{N_{k}}\right\rangle \geq 0 \text {. }
$$

Hence, for all $x \in C$ we have

$$
\langle F x, x-z\rangle=\lim _{k \rightarrow \infty}\left\langle F x, x-v_{N_{k}}\right\rangle=\liminf _{k \rightarrow \infty}\left\langle F x, x-v_{N_{k}}\right\rangle \geq 0 .
$$

By Lemma 2.5 we obtain $z \in \operatorname{Sol}(C, F)$ and the proof is complete.

Remark 3.2. When the function $F$ is monotone, it is not necessary to impose the sequential weak continuity on $F$.

Theorem 3.1. Assume that Conditions 1-3 hold. Then any sequence $\left\{v_{n}\right\}$ generated by Algorithm 2 converges weakly to an element of $\operatorname{Sol}(C, F)$.

Proof. Let $u^{*}$ be a certain solution of (VIP). We divide the proof into two claims.

Claim 1. There exists $N_{1} \in \mathbb{N}$ such that

$$
\left\|v_{n+1}-u^{*}\right\|^{2} \leq\left\|v_{n}-u^{*}\right\|^{2}-(1-\mu)\left\|y_{n}-v_{n}\right\|^{2}-(1-\mu)\left\|v_{n+1}-y_{n}\right\|^{2} .
$$


Extragradient method with a new adaptive step size for solving non-Lipschitzian pseudo-monotone variational inequalitie 1

Indeed, from $u^{*} \in \operatorname{Sol}(\mathrm{C}, \mathrm{F}) \subset \mathrm{C}$, we have

$$
\begin{aligned}
\left\|v_{n+1}-u^{*}\right\|^{2} & =\left\|P_{T_{n}}\left(v_{n}-\tau_{n} F y_{n}\right)-P_{T_{n}} u^{*}\right\|^{2} \leq\left\langle v_{n+1}-u^{*}, v_{n}-\tau_{n} F y_{n}-u^{*}\right\rangle \\
& =\frac{1}{2}\left\|v_{n+1}-u^{*}\right\|^{2}+\frac{1}{2}\left\|v_{n}-\tau_{n} F y_{n}-u^{*}\right\|^{2}-\frac{1}{2}\left\|v_{n+1}-v_{n}+\tau_{n} F y_{n}\right\|^{2} \\
& =\frac{1}{2}\left\|v_{n+1}-u^{*}\right\|^{2}+\frac{1}{2}\left\|v_{n}-u^{*}\right\|^{2}+\frac{1}{2} \tau_{n}^{2}\left\|F y_{n}\right\|^{2}-\left\langle v_{n}-u^{*}, \tau_{n} F y_{n}\right\rangle \\
& -\frac{1}{2}\left\|v_{n+1}-v_{n}\right\|^{2}-\frac{1}{2} \tau_{n}^{2}\left\|F y_{n}\right\|^{2}-\left\langle v_{n+1}-v_{n}, \tau_{n} F y_{n}\right\rangle \\
& =\frac{1}{2}\left\|v_{n+1}-u^{*}\right\|^{2}+\frac{1}{2}\left\|v_{n}-u^{*}\right\|^{2}-\frac{1}{2}\left\|v_{n+1}-v_{n}\right\|^{2}-\left\langle v_{n+1}-u^{*}, \tau_{n} F y_{n}\right\rangle .
\end{aligned}
$$

This implies that

$$
\left\|v_{n+1}-u^{*}\right\|^{2} \leq\left\|v_{n}-u^{*}\right\|^{2}-\left\|v_{n+1}-v_{n}\right\|^{2}-2\left\langle v_{n+1}-u^{*}, \tau_{n} F y_{n}\right\rangle .
$$

Since $u^{*}$ is a solution of the problem (VI), we have $\left\langle F u^{*}, x-u^{*}\right\rangle \geq 0$ for all $x \in C$. By the pseudomontonicity of $F$ on $C$, we have $\left\langle F x, x-u^{*}\right\rangle \geq 0$ for all $x \in C$. Taking $x:=y_{n} \in C$, we get

Thus we have

$$
\left\langle F y_{n}, u^{*}-y_{n}\right\rangle \leq 0
$$

$$
\left\langle F y_{n}, u^{*}-v_{n+1}\right\rangle=\left\langle F y_{n}, u^{*}-y_{n}\right\rangle+\left\langle F y_{n}, y_{n}-v_{n+1}\right\rangle \leq\left\langle F y_{n}, y_{n}-v_{n+1}\right\rangle \text {. }
$$

From (3.20) and (3.21), we obtain

$$
\begin{aligned}
\left\|v_{n+1}-u^{*}\right\|^{2} \leq & \left\|v_{n}-u^{*}\right\|^{2}-\left\|v_{n+1}-v_{n}\right\|^{2}+2 \tau_{n}\left\langle F y_{n}, y_{n}-v_{n+1}\right\rangle \\
= & \left\|v_{n}-u^{*}\right\|^{2}-\left\|v_{n+1}-y_{n}\right\|^{2}-\left\|y_{n}-v_{n}\right\|^{2}-2\left\langle v_{n+1}-y_{n}, y_{n}-v_{n}\right\rangle \\
& +2 \tau_{n}\left\langle F y_{n}, y_{n}-u_{n+1}\right\rangle \\
= & \left\|v_{n}-u^{*}\right\|^{2}-\left\|v_{n+1}-y_{n}\right\|^{2}-\left\|y_{n}-v_{n}\right\|^{2}+2\left\langle v_{n}-\tau_{n} F y_{n}-y_{n}, v_{n+1}-y_{n}\right\rangle .
\end{aligned}
$$

Since $y_{n}=P_{C}\left(v_{n}-\tau_{n} F v_{n}\right)$ and $v_{n+1} \in C$, we have

$2\left\langle v_{n}-\tau_{n} F y_{n}-y_{n}, v_{n+1}-y_{n}\right\rangle=2\left\langle v_{n}-\tau_{n} F v_{n}-y_{n}, v_{n+1}-y_{n}\right\rangle+2 \tau_{n}\left\langle F v_{n}-F y_{n}, v_{n+1}-y_{n}\right\rangle$

$$
\leq 2 \tau_{n}\left\langle F v_{n}-F y_{n}, v_{n+1}-y_{n}\right\rangle .
$$

Combining (3.3) and (3.23), we obtain

$$
2\left\langle v_{n}-\tau_{n} F y_{n}-y_{n}, v_{n+1}-y_{n}\right\rangle \leq \mu\left\|v_{n}-y_{n}\right\|^{2}+\mu\left\|y_{n}-v_{n+1}\right\|^{2} .
$$

Substituting (3.24) into (3.22), we obtain

$$
\left\|v_{n+1}-u^{*}\right\|^{2} \leq\left\|v_{n}-u^{*}\right\|^{2}-(1-\mu)\left\|y_{n}-v_{n}\right\|^{2}-(1-\mu)\left\|v_{n+1}-y_{n}\right\|^{2} .
$$

Claim 2. Now, we show that $\left\{v_{n}\right\}$ converges weakly to an element of $\operatorname{Sol}(C, F)$. Thanks to Claim 1 we have

$$
\left\|v_{n+1}-u^{*}\right\| \leq\left\|v_{n}-u^{*}\right\| .
$$

This implies that for all $u^{*} \in \operatorname{Sol}(C, F)$ then $\lim _{n \rightarrow \infty}\left\|v_{n}-u^{*}\right\|$ exists, thus the sequence $\left\{v_{n}\right\}$ is bounded. Consequently, $\left\{v_{n}\right\}$ is bounded.

On the other hand, according to Claim 1, we get

$$
(1-\mu)\left\|v_{n}-y_{n}\right\|^{2} \leq\left\|v_{n}-u^{*}\right\|^{2}-\left\|v_{n+1}-u^{*}\right\|^{2}
$$

which implies that

$$
\lim _{n \rightarrow \infty}\left\|v_{n}-y_{n}\right\|=0
$$

Since $\left\{v_{n}\right\}$ is a bounded sequence, there exists the subsequence $\left\{v_{n_{k}}\right\}$ of $\left\{v_{n}\right\}$ such that $\left\{v_{n_{k}}\right\}$ converges weakly to $z \in C$. It implies from Lemma 3.8 and (3.25) that $z \in \operatorname{Sol}(C, F)$. 
Therefore, we showed that:

i) For every $u^{*} \in \operatorname{Sol}(C, A)$, then $\lim _{n \rightarrow \infty}\left\|v_{n}-u^{*}\right\|$ exists;

ii) Every sequential weak cluster point of the sequence $\left\{v_{n}\right\}$ is in $\operatorname{Sol}(C, F)$.

By Lemma 2.6 the sequence $\left\{v_{n}\right\}$ converges weakly to an element of $\operatorname{Sol}(C, F)$.

Remark 3.3. Our results are more general some related results in the literature and hence might be applied for a wider class of mappings. For example, we next present the advantage of our method compared with the recent result [31, Theorem 3.1].

1. In Theorem 3.1, $F: C \rightarrow H$ is assumed to be uniformly continuous on bounded subsets instead of Lipschitz continuous in [31].

2. Note that in our work we use condition (3.4), which is strictly weaker than the sequential weak continuity of the operator $F$, an assumption which has frequently been used in recent articles $[2,31]$ on pseudomonotone variaional inequality problems.

3.2. Convergence rate. In this section, we provide a result on the convergence rate of the iterative sequence generated by Algorithm 2.

Theorem 3.2. Assume that $F: C \rightarrow H$ is L-Lipschitz continuous and $\delta$-strongly pseudomonotone on $C$. Then the sequence $\left\{x_{n}\right\}$ generated by Algorithm 2 converges strongly to $u^{*}$ with a $Q$ - linear rate, where $u^{*}$ is a unique solution of VI.

Proof. We have $\left\langle F u^{*}, y_{n}-u^{*}\right\rangle \geq 0$, by the strong pseudomonotonicity of $F$ on $C$ we get

$$
\left\langle F y_{n}, y_{n}-u^{*}\right\rangle \geq \delta\left\|y_{n}-u^{*}\right\|^{2} \text {. }
$$

Using (3.26), we have

$\left\langle F y_{n}, u^{*}-v_{n+1}\right\rangle=\left\langle F y_{n}, u^{*}-y_{n}\right\rangle+\left\langle F y_{n}, y_{n}-v_{n+1}\right\rangle \leq-\delta\left\|y_{n}-u^{*}\right\|^{2}+\left\langle F y_{n}, y_{n}-v_{n+1}\right\rangle$.

Substituting (3.27) into (3.20), we obtain

$$
\left\|v_{n+1}-u^{*}\right\|^{2} \leq\left\|v_{n}-u^{*}\right\|^{2}-\left\|v_{n+1}-v_{n}\right\|^{2}-2 \tau_{n} \delta\left\|y_{n}-u^{*}\right\|^{2}+2 \tau_{n}\left\langle F y_{n}, y_{n}-v_{n+1}\right\rangle .
$$

Thus

$$
\begin{aligned}
\left\|v_{n+1}-u^{*}\right\|^{2} \leq & \left\|v_{n}-u^{*}\right\|^{2}-\left\|v_{n+1}-v_{n}\right\|^{2}-2 \tau_{n} \delta\left\|y_{n}-u^{*}\right\|^{2}+2 \tau_{n}\left\langle F y_{n}, y_{n}-v_{n+1}\right\rangle \\
= & \left\|v_{n}-u^{*}\right\|^{2}-\left\|v_{n+1}-y_{n}\right\|^{2}-\left\|y_{n}-v_{n}\right\|^{2}-2\left\langle v_{n+1}-y_{n}, y_{n}-v_{n}\right\rangle \\
& -2 \tau_{n} \delta\left\|y_{n}-u^{*}\right\|^{2}+2 \tau_{n}\left\langle F y_{n}, y_{n}-v_{n+1}\right\rangle \\
= & \left\|v_{n}-u^{*}\right\|^{2}-\left\|v_{n+1}-y_{n}\right\|^{2}-\left\|y_{n}-v_{n}\right\|^{2}-2 \tau_{n} \delta\left\|y_{n}-u^{*}\right\|^{2} \\
& +2\left\langle v_{n}-\tau_{n} F y_{n}-y_{n}, v_{n+1}-y_{n}\right\rangle .
\end{aligned}
$$

Substituting (3.24) into (3.28), we obtain

$\left\|v_{n+1}-u^{*}\right\|^{2} \leq\left\|v_{n}-u^{*}\right\|^{2}-(1-\mu)\left\|y_{n}-v_{n}\right\|^{2}-(1-\mu)\left\|v_{n+1}-y_{n}\right\|^{2}-2 \tau_{n} \delta\left\|y_{n}-u^{*}\right\|^{2}$.

Now we show that $\tau_{n}>\frac{\mu l}{L}$ for all $n$. Indeed, by the search rule (3.3), we know that $\frac{\tau_{n}}{l}$ must violate inequality (3.3), i.e.,

$$
\left\langle F y_{n}-F v_{n}, y_{n}-v_{n+1}\right\rangle>\frac{\mu}{2 \frac{\tau_{n}}{l}}\left(\left\|v_{n}-y_{n}\right\|^{2}+\left\|y_{n}-v_{n+1}\right\|^{2}\right) .
$$


This follows that

$$
L\left\|y_{n}-v_{n}\right\|\left\|y_{n}-v_{n+1}\right\|>\frac{\mu}{2 \frac{\tau_{n}}{l}}\left(\left\|v_{n}-y_{n}\right\|^{2}+\left\|y_{n}-v_{n+1}\right\|^{2}\right) .
$$

Thus

$$
\frac{L}{2}\left(\left\|y_{n}-v_{n}\right\|^{2}+\left\|y_{n}-v_{n+1}\right\|^{2}\right)>\frac{\mu}{2 \frac{\tau_{n}}{l}}\left(\left\|v_{n}-y_{n}\right\|^{2}+\left\|y_{n}-v_{n+1}\right\|^{2}\right) .
$$

This implies that

$$
\tau_{n}>\frac{\mu l}{L} \forall n
$$

Combining (3.29) and (3.30) we get

$$
\begin{aligned}
& \left\|v_{n+1}-u^{*}\right\|^{2} \leq\left\|v_{n}-u^{*}\right\|^{2}-(1-\mu)\left\|y_{n}-v_{n}\right\|^{2}-(1-\mu)\left\|v_{n+1}-y_{n}\right\|^{2}-2 \frac{\mu l}{L} \delta\left\|y_{n}-u^{*}\right\|^{2} \\
& \quad \leq\left\|v_{n}-u^{*}\right\|^{2}-(1-\mu)\left\|y_{n}-v_{n}\right\|^{2}-2 \frac{\mu l}{L} \delta\left\|y_{n}-u^{*}\right\|^{2} .
\end{aligned}
$$

Let $\beta:=\min \left\{\frac{1-\mu}{2}, \frac{\mu l}{L} \delta\right\}$. From (3.31), we get

$$
\begin{aligned}
\left\|v_{n+1}-u^{*}\right\|^{2} & \leq\left\|v_{n}-u^{*}\right\|^{2}-2 \beta\left\|y_{n}-v_{n}\right\|^{2}-2 \beta\left\|y_{n}-u^{*}\right\|^{2} \\
& \leq\left\|v_{n}-u^{*}\right\|^{2}-\beta\left\|v_{n}-u^{*}\right\|^{2} \\
& =(1-\beta)\left\|v_{n}-u^{*}\right\|^{2} .
\end{aligned}
$$

This implies that

$$
\left\|v_{n+1}-u^{*}\right\| \leq \sqrt{(1-\beta)}\left\|v_{n}-u^{*}\right\| .
$$

Since $\sqrt{(1-\beta)} \in(0,1)$, it implies that the sequence $\left\{v_{n}\right\}$ converges strongly to $u^{*}$ with a Q-linear rate.

\section{NUMERICAL ILLUSTRATIONS}

In this section, we perform numerical experiments to show the behaviors of Algorithm 2 and compare it with other algorithms. All the numerical experiments are performed on an HP laptop with Intel(R) Core(TM)i5-6200U CPU 2.3GHz with 4 GB RAM. All the programs are written in Matlab2015a. The followings are the experiment in detail. Example 4.1 Let $F(x):=M x+q$ where

$$
M=B B^{T}+C+D,
$$

and $B$ is an $m \times m$ matrix, $C$ is an $m \times m$ skew-symmetric matrix, $D$ is an $m \times m$ diagonal matrix, whose diagonal entries are nonnegative (so $M$ is positive semidefinite), $q$ is a vector in $\mathbb{R}^{m}$. The feasible set $C \subset \mathbb{R}^{m}$ is a box constraints in $\mathbb{R}^{m}$ defined by

$$
C:=\left\{x \in \mathbb{R}^{m}: 0 \leq x \leq 1\right\} .
$$

It is clear that $F$ is monotone and Lipschitz-continuous with constant $L=\|M\|$. Let $q=0$. Then, we obtain the solution set $\Gamma=\{0\}$. The parameters are chosen as follows:

Algorithm 2: $\gamma=0.01, \mu=0.5, l=0.5, \beta_{n}=\frac{1}{n+2}$

Algorithm 3.1 in [2]: $f(x)=0.5 x, \gamma=0.01, \mu=0.5, l=0.5, \beta_{n}=\frac{1}{n+2}$.

Algorithm 3.2 in [13]: $f(x)=0.5 x, \lambda=0.01, \gamma=1, \mu=0.5, l=0.5, \beta_{n}=\frac{1}{n+2}$.

Algorithm 3.1 in [30]: $\tau_{0}=0.001, \mu=0.5, \alpha_{n}=\frac{1}{n+2}, \beta_{n}=0.99\left(1-\alpha_{n}\right)$.

For experiment, all entries of $B, C$ and $D$ are generated randomly from a normal distribution with mean zero and unit variance. We use stopping rule $\left\|x_{n}\right\|<10^{-7}$ or 
Iter $\geq 1000$ for all algorithms. The numerical results are described in Table 1 and Fig. 1, Fig. 2.

TABLE 1. Numerical results obtained by other algorithms

\begin{tabular}{cccccccc}
\hline \multirow{2}{*}{ Methods } & \multicolumn{3}{c}{$\mathrm{m}=100$} & & \multicolumn{3}{c}{$\mathrm{m}=200$} \\
\cline { 2 - 4 } \cline { 7 - 8 } & Sec. & Iter. & Error. & & Sec. & Iter. & Error. \\
\hline Algorithm 2 & 0.04 & 82 & $8.6284 \mathrm{e}-08$ & & 0.0980 & 75 & $9.3728 \mathrm{e}-08$ \\
\hline Algorithm 3.1 in [2] & 1.016 & 1000 & 0.0014 & & 3.8010 & 1000 & 0.0023 \\
\hline Algorithm 3.2 in [13] & 0.2410 & 777 & $9.9722 \mathrm{e}-08$ & & 1.5350 & 1000 & $2.2974 \mathrm{e}-07$ \\
\hline Algorithm 3.1 in [30] & 0.1470 & 1000 & $4.8767 \mathrm{e}-04$ & & 0.4960 & 1000 & $5.6404 \mathrm{e}-04$ \\
\hline
\end{tabular}

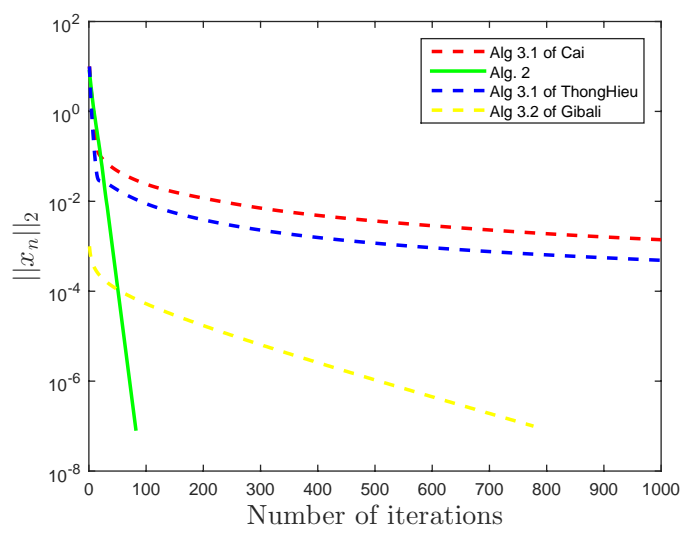

FIGURE 1. Comparison of all algorithms with $m=100$

Fig. 1-2 and Table 1 demonstrate that Algorithm 2 performs better than Algorithm 3.1 in [2], Algorithm 3.2 in [13] and Algorithm 3.1 in [30].

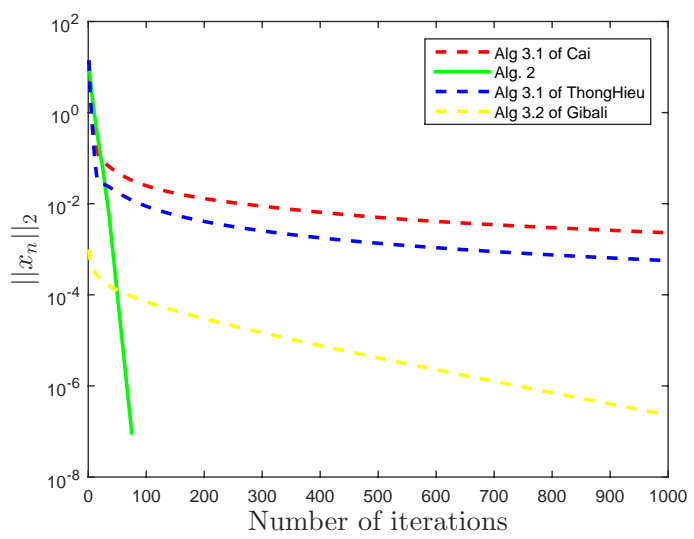

FIGURE 2. Com parison of all algorithms with $m=200$ 


\section{CONCLUSIONS}

In this paper we proposed a modification of extragradient method for solving nonLipschitzian pseudo-monotone variational inequalities in real Hilbert spaces. Under suitable conditions we establish weak convergence and convergence rate of the proposed scheme. Our work extend and generalize some existing results in the literature.

\section{REFERENCES}

[1] Antipin, A. S. On a method for convex programs using a symmetrical modification of the Lagrange function. Ekonomika i Mat. Metody. 12 (1976), 1164-1173

[2] Cai, G.; Dong, Q. L.; Peng, Y. Strong convergence theorems for solving variational inequality problems with pseudo-monotone and non-Lipschitz operators. J. Optim. Theory Appl. (2021). https:/ / doi.org/10.1007/s10957-020-01792-w

[3] Censor, Y.; Gibali, A.; Reich, S. The subgradient extragradientmethod for solving variational inequalities in Hilbert space. J. Optim. Theory Appl. 148 (2011), 318-335.

[4] Censor, Y.; Gibali, A.; Reich, S. Strong convergence of subgradient extragradient methods for the variational inequality problem in Hilbert space. Optim. Meth. Softw. 26 (2011), 827-845.

[5] Censor, Y.; Gibali, A.; Reich, S. Extensions of Korpelevich's extragradient method for the variational inequality problem in Euclidean space. Optimization 61 (2011), 1119-1132.

[6] Censor, Y.; Gibali, A.; Reich, S. Algorithms for the split variational inequality problem. Numer. Algorithms. 56 (2012), 301-323.

[7] Cottle, R. W.; Yao, J. C. Pseudo-monotone complementarity problems in Hilbert space. J. Optim. Theory Appl. 75 (1992), 281-295.

[8] Denisov, S. V.; Semenov, V. V.; Chabak, L. M. Convergence of the modified extragradient method for variational inequalities with non-Lipschitz operators. Cybern. Syst. Anal. 51 (2015), 757-765.

[9] Facchinei, F.; Pang, J. S. Finite-Dimensional Variational Inequalities and Complementarity Problems. Springer Series in Operations Research, vols. I and II. Springer, New York 2003.

[10] Fichera, G.: Sul problema elastostatico di Signorini con ambigue condizioni al contorno. Atti Accad. Naz. Lincei, VIII. Ser., Rend., Cl. Sci. Fis. Mat. Nat. 34 (1963), 138-142.

[11] Fichera, G. Problemi elastostatici con vincoli unilaterali: il problema di Signorini con ambigue condizioni al contorno. Atti Accad. Naz. Lincei, Mem., Cl. Sci. Fis. Mat. Nat., Sez. I, VIII. Ser. 7 (1964), 91-140 .

[12] Gibali, A. A new non-Lipschitzian projection method for solving variational inequalities in Euclidean spaces. Journal of Nonlinear Analysis and Optimization: Theory and Applications 6 (2015), 41-51.

[13] Gibali, A.; Thong, D. V.; Tuan, P. A. Two simple projection-type methods for solving variational inequalities. Anal. Math. Phys. 9 (2019), 220-2225.

[14] Goebel, K.; Reich, S. Uniform Convexity, Hyperbolic Geometry and Nonexpansive Mappings. Marcel Dekker, New York 1984

[15] Ha, N. T. T.; Thanh, T. T. H.; Hai, N. N. et al. A note on the combination of equilibrium problems. Math. Meth. Oper. Res. 91 (2020), 311-323.

[16] Iusem, A. N. An iterative algorithm for the variational inequality problem. Comput. Appl. Math. 13 (1994), 103-114.

[17] Iusem, A. N.; Nasri, M. Korpelevich's method for variational inequality problems in Banach spaces, J. Glob. Optim. 50 (2011), 59-76.

[18] Karamardian, S. Complementarity problems over cones with monotone and pseudo-monotone maps. J. Optim. Theory Appl. 18 (1976), 445-454.

[19] Khobotov, E. N. Modifications of the extragradient method for solving variational inequalities and certain optimization problems. USSR Computational Mathematics and Mathematical Physics. 27 (1987), 120-127.

[20] Korpelevich, G. M. The extragradient method for finding saddle points and other problems. Ekonomika $i$ Mat. Metody. 12 (1976), 747-756.

[21] Konnov, I. V. Equilibrium formulations of relative optimization problems. Math. Meth. Oper. Res. 90 (2019), 137-152.

[22] Konnov, I. V. A general class of relative optimization problems. Math. Meth. Oper. Res. (2021). https://doi.org/10.1007/s00186-021-00741-1.

[23] Malitsky, Y. V. Projected reflected gradient methods for monotone variational inequalities. SIAM J. Optim. 25 (2015), 502-520

[24] Malitsky, Y. V.; Semenov, V. V. A hybrid method without extrapolation step for solving variational inequality problems. J. Glob. Optim. 61 (2015), 193-202 
[25] Marcotte, P. Application of Khobotov's algorithm to variational inequalities and network equilibrium problems. Information Systems and Operational Research. 29 (1991), 258-270

[26] Opial, Z. Weak convergence of the sequence of successive approximations for nonexpansive mappings. Bulletin of the American Mathematical Society 73 (1967), 591-597

[27] Ortega, J. M.; Rheinboldt, W. C. Iterative Solution of Nonlinear Equations in Several Variables. Academic Press, New York (1970)

[28] Shehu, Y.; Iyiola, O. S.; Thong, D. V.; Van, N. T. C. An inertial subgradient extragradient algorithm extended to pseudomonotone equilibrium problems. Math. Meth. Oper. Res. (2020) https://doi.org/10.1007/s00186020-00730-w

[29] Solodov, M. V.; Svaiter, B. F. A new projection method for variational inequality problems. SIAM J. Control Optim. 37 (1999), 765-776

[30] Thong, D. V.; Hieu, D. V.; Rassias, T. M. Self adaptive inertial subgradient extragradient algorithms for solving pseudomonotone variational inequality problems. Optimiz. Lett. 14 (2020), 115-144

[31] Vuong, P. T. On the weak convergence of the extragradient method for solving pseudo-monotone variational inequalities. J. Optim. Theory Appl. 176 (2018), 399-409

Division of ApPlied MATHEMATiCs

Thu Dau Mot University, Binh Duong Province, Vietnam

Email address: duongviet hongetdmu.edu.vn 